\title{
De Novo Urinary Symptoms Associated With COVID-19: COVID-19-Associated Cystitis
}

\author{
Nivedita Dhar ${ }^{\mathrm{a}, \mathrm{b}}$, Sorabh Dhar ${ }^{\mathrm{b}, \mathrm{c}}$, Ryan Timar ${ }^{\mathrm{c}}$, Steven Lucas ${ }^{\mathrm{b}, \mathrm{c}}$, \\ Laura E. Lamb ${ }^{\mathrm{d}, ~ e}$, Michael B. Chancellor ${ }^{\mathrm{d}, \mathrm{e}, \mathrm{f}}$
}

\section{To the Editor}

Clinical symptoms that present early in coronavirus disease 2019 (COVID-19), which is caused by severe acute respiratory syndrome coronavirus 2 (SARS-CoV-2), have been well-reported. Although the majority number of patients develop mild symptoms, a small percentage can progressively develop acute respiratory distress syndrome and multiple organ dysfunction syndrome resulting in demise [1]. There is an emergence of new symptoms that involve many organ systems, some more subtle than others. As these symptoms may overlap with other common disease processes, it has been difficult to identify and link these symptoms as direct association with COVID-19 as the underlying cause [2].

Recently Mumm and his colleagues have reported increased urinary frequency in COVID-19 patients [3]. We noticed at our tertiary care medical center's COVID-19 clinic that patients reported de novo urinary tract symptoms and therefore obtained institutional review board (IRB) approval for a case series. We surveyed urinary symptoms in patients who followed up in the outpatient setting after their hospitalization from COVID-19. All patients had confirmed positive SARS-CoV-2 molecular diagnostic test. Patients filled out their survey responses in the office setting. Urinary symptoms are based on a validated bladder health questionnaire (Overactive Bladder (OAB) Assessment Tool) [4]: The five individual symptom scores range from 0 to 5 based on urinary urgency, urge incontinence, incontinence, frequency, and nocturia. We noted patient's hospital dates to establish length-of-stay (LOS) and the study was conducted from May 22 to June 26, 2020.

We identified 39 COVID-19-positive patients, including seven females and 32 males, who developed de novo urinary symptoms without urinary tract infection per standard urine

Manuscript submitted July 21, 2020, accepted July 27, 2020

Published online September 21, 2020

aDetroit Medical Center, Detroit, MI, USA

bJohn D. Dingell VA Medical Center, Detroit, MI, USA

${ }^{\mathrm{c}}$ Wayne State University School of Medicine, Detroit, MI, USA

dDepartment of Urology, Beaumont Health System, Royal Oak, MI, USA

'Oakland University William Beaumont School of Medicine, Rochester Hills, MI, USA

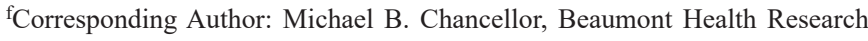
Institute, 3811 W. 13 Mile Road, Suite 504, Royal Oak, MI 48073, USA. Email: Michael.Chancellor@Beaumont.org

doi: https://doi.org/10.14740/jocmr4294 culture and sensitivity testing. The patients were all outpatients post hospital discharge and did not have fever or other clinical conditions that would require inpatient health care. All patients were African American. Median LOS was 10 days (range 5 30). All 39 patients completed the symptom score survey. The median total $\mathrm{OAB}$ symptom score in men and women was 18 (ranges $12-20$ and $15-21$, respectively) (Table 1). Our case series bring awareness to the possibility of new onset urinary symptoms in COVID-19 patients and the cause of the symptoms remains unsolved. The most remarkable urologic complaints were increased urinary frequency of $\geq 13$ episodes $/ 24 \mathrm{~h}$ $(85 \%)$ and nocturia $\geq$ four episodes/night $(87 \%)$.

In conclusion, in a limited survey of patients at a tertiary care COVID-19 clinic, we found COVID-19 patients, both men and women, may report de novo lower urinary tract symptoms. The most bothersome new urinary tract symptoms include urinary frequency and nocturia. Physicians caring for COVID-19 should be aware of COVID-19-associated cystitis (CAC).

\section{Acknowledgments}

None to declare.

\section{Financial Disclosure}

None to declare.

\section{Conflict of Interest}

The authors declare that they have no relevant conflict of interest to report.

\section{Informed Consent}

Informed consents have been obtained from the patients.

\section{Author Contributions}

MC and ND contributed to the formulation. SD, ND, LL, and MC planned the study. RT, ML, ND, and SD collected the data. 
Table 1. Demographics and Symptoms

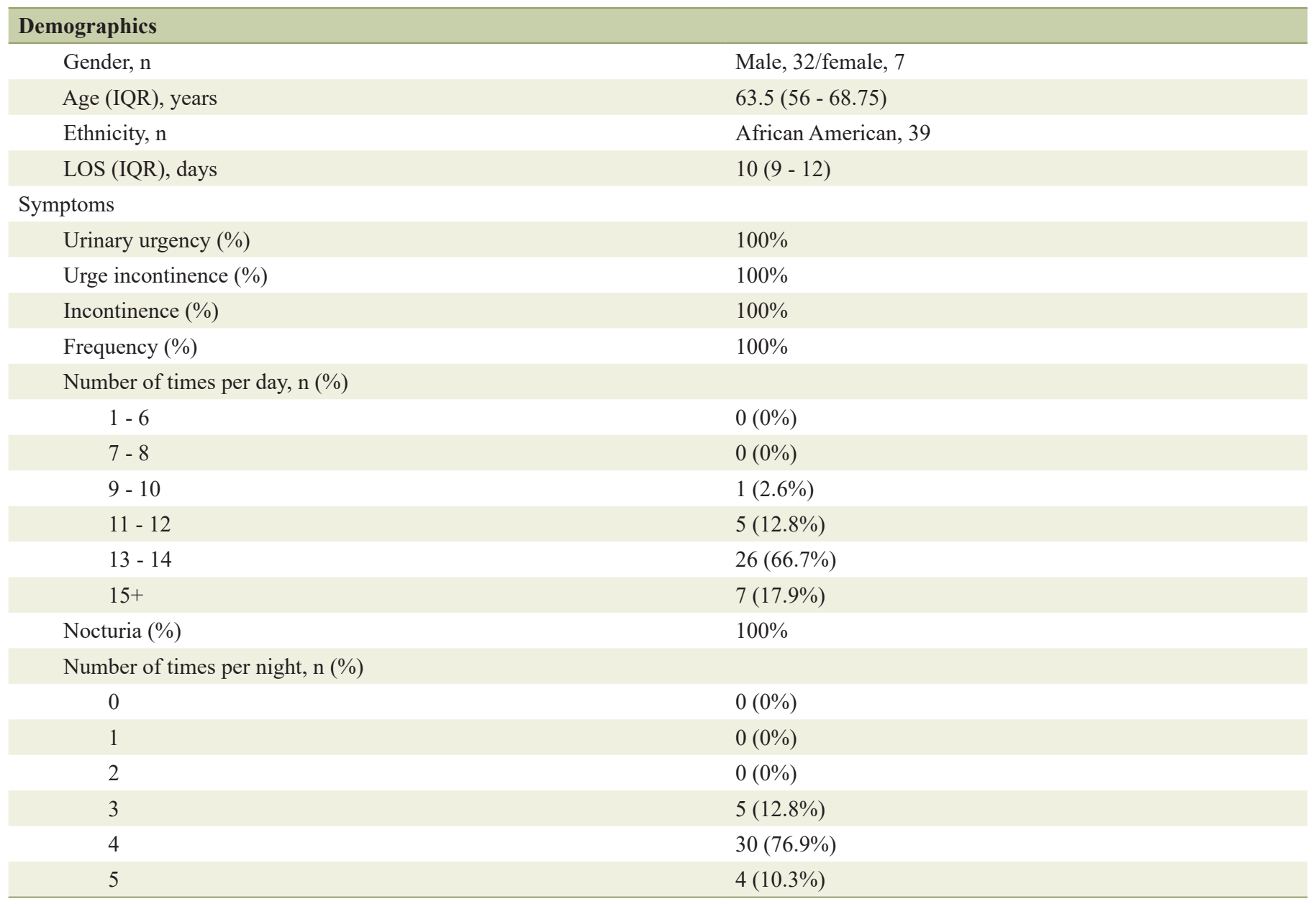

IQR: interquartile range; LOS: length-of-stay.

LL, MC, ND, and SD analyzed the data. All authors involved in writing the manuscript.

\section{Data Availability}

The data supporting the findings of this study are available from the corresponding author upon reasonable request.

\section{References}

1. Wang H, Ma S. The cytokine storm and factors determin- ing the sequence and severity of organ dysfunction in multiple organ dysfunction syndrome. Am J Emerg Med. 2008;26(6):711-715.

2. Sighinolfi MC, Rocco B, Mussini C. COVID-19: Importance of the Awareness of the Clinical Syndrome by Urologists. Eur Urol. 2020;78(1):e40-e41.

3. Mumm JN, Osterman A, Ruzicka M, Stihl C, Vilsmaier T, Munker D, Khatamzas E, et al. Urinary Frequency as a Possibly Overlooked Symptom in COVID-19 Patients: Does SARS-CoV-2 Cause Viral Cystitis? Eur Urol. 2020.

4. Overactive Bladder Assessment Tool. 2018. https://www. urologyhealth.org/educational-materials/overactive-bladder-assessment-tool-x4801. Accessed May 1, 2020. 